\title{
Las competencias digitales docentes en entornos universitarios basados en el Digcomp
}

\section{Competências digitais de docentes em ambientes universitários baseados no Digcomp}

\section{Teachers digital competences in Digcomp-based university environments}

\author{
Ana María Martín Cuadrado* \\ Lourdes Pérez Sánchez* \\ María Jordano de la Torre*
}

\begin{abstract}
RESUMEN
Este artículo describe una propuesta de adaptación de los descriptores sugeridos por la Unión Europea mediante el DigComp para las áreas competenciales de la Información y la Comunicación al personal docente universitario. Esta se ha llevado a cabo gracias a la retroalimentación recibida de diferentes cursos impartidos en forma de NOOC (Nano-Open Massive Courses) en diferentes ediciones desde el año académico 2016-17. De este modo, se han obtenido datos provenientes de los test de diagnóstico realizados antes de realizar cada curso, encuestas iniciales y finales a cada usuario y la propia observación de los mismos. Una de las conclusiones más relevantes del estudio ha sido la del haber recibido más cantidad de alumnos en los cursos de nivel básico, seguida del avanzado y del intermedio, en la mayoría de los casos. El estudio que aquí se presenta se centra, entre otras cosas, en tratar de encontrar las razones por las cuales se ha producido este
\end{abstract}

*Universidad Nacional de Educación a Distancia. Madrid, España. E-mail: amartin@edu. uned.es - https://orcid.org/0000-0001-7579-3701 E-mail: 1perezsanchez@edu.uned.es - https:// orcid.org/0000-0002-9767-4639 E-mail: mjordano@flog.uned.es - https://orcid.org/0000-00017779-9584 
hecho a la vez que se muestra una panorámica del grado de competencia digital con el que cuentan estos docentes en la actualidad. Esto nos permitirá seguir adaptando los descriptores anteriormente mencionados a las necesidades actuales, que incluso podrían haberse visto influenciadas por la crisis del Covid-19 que provocó que la enseñanza presencial se digitalizara en un corto periodo de tiempo.

Palabras clave: Formación docente. Trabajo docente. Competencias digitales. Nanocursos. Microcredenciales.

\title{
RESUMO
}

Este artigo descreve uma proposta para adaptar os descritores sugeridos pela União Europeia através do DigComp para as áreas de habilidades de informação e comunicação para professores universitários. O estudo foi realizado graças ao feedback recebido de diferentes cursos ofertados na forma de NOOC (Nano-Open Massive Courses) em diferentes edições de 2016-17. Os dados foram obtidos apartir dos testes de diagnóstico realizados no inicio de cada curso e finais de cada usuário apartir de sua própria observação. Uma das conclusões mais relevantes do estudo foi a de ter recebido mais alunos em cursos de nível básico, seguidos de avançado e intermediário. O estudo apresentado aqui concentra-se, entre outras coisas, em tentar encontrar as razões pelas quais isso ocorreu, mostrando uma visão geral do grau de competência digital que esses professores possuem. Isso nos permitirá continuar adaptando e atualizando os descritores mencionados às necessidades atuais. Destaca-se que os resultados podem ter sido influenciados pela crise do Covid-19 que obrigou a digitalização das atividades de ensino em sala de aula em um curto período de tempo.

Palavras-chave: Formação docente. Trabalho docente. Habilidades digitais. Nanocursos. Micro credenciais.

\begin{abstract}
This article discusses a proposal to adapt the descriptors suggested by the European Union through DigComp for the areas of information and communication skills for university professors. The study was carried out thanks to the feedback received from different courses offered in the form of NOOC (Nano-Open Massive Courses) in different editions in 2016 and 2017. The data were obtained from diagnostic tests performed at the beginning and at the end of each course by the user, from their own observation. One of the most relevant conclusions of the study was that it had more students in basic level courses, followed by advanced and intermediate. The study presented here focuses, among other things, on trying to find the reasons why this occurred, showing an overview of the degree of digital competence that
\end{abstract}


these teachers have. This will allow us to continue adapting and updating the mentioned descriptors to current needs. It is noteworthy that the results may have been influenced by the Covid-19 crisis; which forced the digitalization of classroom teaching activities in a short period.

Keywords: Teacher training. Higher education teaching. Digital competences. NOOCs. Micro-credentials.

\section{Introducción}

Desde que saliera a la luz la primera versión del Marco de Competencia Digital Europeo, en adelante DigComp (FERRARI, 2013) fruto de la inclusión de las competencias digitales como una de las ocho descritas 2006 por el Parlamento Europeo, no han dejado de aportarse actualizaciones. Esta proponía cinco áreas, basadas a su vez en otro documento anterior elaborado por la UNESCO (UNITED NATIONS, 2011), eran: Información, Comunicación, Creación de contenido, Seguridad y Resolución de problemas. Además, contenían un total de 21 competencias correspondientes, y tres niveles de desempeño por cada una (inicial, medio y avanzado). La primera actualización no sólo trae consigo un cambio de denominación de algunas de las áreas, sino que se incluyen ejemplos de uso en diferentes países europeos -entre ellos, España- y un listo de conceptos clave (VUORIKARI et al., 2016). La siguiente versión incorpora cuatro niveles de desempeño en lugar de tres, que se subdividen a su vez en dos adicionales haciendo un total de ocho (CARRETERO; VUORIKARI; PUNIE, 2017a). En la actualidad, están sirviendo de base para ser adaptados a diferentes contextos profesionales, entre ellos el educativo (REDECKER, 2017). La propuesta formativa a continuación se presenta adaptada a docentes universitarios y se ha basado, principalmente, en la segunda versión formativa del DigComp (VUORIKARI; PUNIE, 2016), ya que cada competencia abarca tres niveles de dificultad, dejando el cuarto de la edición posterior para formar parte de la certificación del área competencial (JORDANO DE LA TORRE; MARTÍN-CUADRADO; PÉREZ SÁNCHEZ, 2019). Por otro lado, aunque la versión educativa del DigComp se ha tenido en cuenta, no se ha seguido totalmente, ya que la figura del profesor universitario va más allá de las puramente docentes (EUROPEAN COMMISSION/EACEA/EURYDICE, 2017; MONEREO; DOMÍNGUEZ, 2014) en primer lugar, de validar el modelo de perfil de competencias del docente universitario, elaborado recientemente por ocho universidades catalanas (Torra et al., 2012). De ahí la necesidad de adaptar 
los descriptores de cada competencia a las necesidades presentes en cualquier profesor universitario, como la investigadora y de gestión (PÉREZ SÁNCHEZ; JORDANO DE LA TORRE; MARTÍN CUADRADO, 2017).

De las cinco áreas competenciales que consta el DigComp que se ha tomado como base, este artículo se ha centrado en las dos primeras (Información y Alfabetización informacional y Comunicación y Colaboración); las tres restantes se irán trabajando en ediciones posteriores. Así la primera área competencial del DigComp, en adelante NOOC1, ha terminado su cuarta edición en diciembre de 2019 y la primera edición de la segunda área, en adelante NOOC2, se ha presentado en enero de 2020. Entre medias, y fruto de la emergencia provocada por el Covid-19, se han replicado versiones especiales para la primera y segunda competencia de los NOOC2 (Interacción a través de las tecnologías; Compartir información y contenido) de forma que sirvan a otros profesores de la universidad presencial a adaptar sus contenidos a entornos a distancia mediante la iniciativa de \#yomeformoencasa ${ }^{1}$.

Como se ha comentado anteriormente, de una edición a otra se van incorporando cambios y mejoras, muchas de ellas sustanciales, como la que se ha llevado a cabo en la cuarta edición de los NOOC1.Esta incluye la acreditación de sus tres competencias por separado, mediante tres cursos diseñados ad hoc, y una certificación final del área competencial con la realización de un trabajo de investigación que demuestre la adquisición de las tres competencias en los diferentes roles del profesor universitario (JORDANO DE LA TORRE; MARTÍN CUADRADO; PÉREZ SÁNCHEZ, 2019).

En consonancia con otros autores (DURÁN CUARTERO; GUTIÉRREZ PORLÁN, 2016), consideramos de vital importancia validar y certificar la competencia digital del docente universitario en todos sus entornos: docencia, investigación y gestión.

A partir del NOOC2 se han incorporado al equipo de trabajo otros investigadores y personal docente de diferentes universidades españolas y brasileñas, con la idea de ir sumando más investigadores y profesionales para la elaboración de otros cursos en las áreas competenciales sucesivas. Además, fruto de los datos obtenidos con esta experiencia, se está participando en diversas actuaciones de investigación: Proyecto Europeo Erasmus+ (Capacity Bulding)²,

1 yomeformoencasa con UNED Abierta https://blogs.uned.es/unedabierta/yomeformoencasa/. La Universidad en casa https://www.uned.es/universidad/inicio/uned_uoc_solidaria.html.

2 Proyecto GECKO (Pr. N. 585934-EPP-1-2017-1-FR-EPPKA2-CBHE-JP). 
Proyecto de Innovación Docente 3 , Proyecto de Investigación PI $1543^{4}$ (ÁLVAREZ et al., 2019) Proyecto Intercoonecta-AECID ${ }^{5}$ (capacitación de formadores en competencias digitales en la región de América Latina y El Caribe).

El objetivo de los cursos que se analizan en este estudio es tratar de ofrecer a los docentes universitarios un apoyo en su propia formación, que sea a su vez capacitadora en el ámbito digital, que es cada vez más necesaria de cara una enseñanza de calidad acorde con el contexto universitario en el que nos encontramos. Ser personal docente e investigador en el siglo XXI requiere, entre otras cosas, ser competente digitalmente. La adquisición y entrenamiento de un conjunto de habilidades, conocimientos y actitudes que se incluyen en la competencia digital deberían facilitar la funcionalidad y operatividad de las actuaciones del docente. De este modo, no sólo se desarrollaría la capacidad de manejar herramientas tecnológicas y dispositivos que van cambiando cada cierto tiempo, sino que las adaptaría de acuerdo a las necesidades de su campo de conocimiento específico (PÉREZ SÁNCHEZ; JORDANO DE LA TORRE; MARTÍN CUADRADO, 2017).

\section{La figura del profesor universitario del siglo XXI}

Hasta hace unas décadas, el principal cometido del profesor universitario consistía en impartir clases magistrales en un aula considerablemente voluminosa, ayudado de sus apuntes, fotocopias que pedía a sus alumnos que recogiera en reprografía y sin más apoyo que el encerado (DE VINCENZI, 2012; MAUREL; CUENCA-PLETSCH; DALFARO, 2013; MONEREO; DOMÍNGUEZ, 2014) Hoy en día, los apuntes se ponen a disposición del alumno en una plataforma digital, al igual que las fotocopias, y la pizarra ha sido reemplazada por una presentación de diapositivas o por la pizarra digital. Además de sus funciones como docente, debe seguir desempeñando otras relacionadas

3 Red de Innovación docente aprobada (curso 2019-20): GID2016-33 Digital TANDEMImproving the L2 Linguistic Competence through technology (UNED-Loughborough University) DiTaUNED_lboro.

4 Participantes: Universidad Nacional de La Patagonia Juan Bosco UNPSJB (Argentina), UNED (España) y Universidad de Sevilla. Aval académico, Resolución R UNPSJB. Periodo de ejecución 01/01/2019-31/12/2020. 
con la investigación (BUSTOS-GONZÁLEZ, 2019) colaborando en grupos de investigación, solicitando tramos de antigüedad, o asistiendo a congresos, entre otras acciones- o la gestión. Para todas estas tareas mencionadas es necesario el poseer un mí mínimo de competencia digital que se van complicando a medida que avanza la tecnología. Aunque existen cursos propuestos por diferentes universidades por formar a los nuevos profesionales (WELLER, 2019), otros optan por reciclarse de manera autónoma, a diferencia de otros que, debido a la rama en la que se mueven, necesitan tiempo y dedicación para ponerse al día en la nueva forma de realizar cada tarea (RODRÍGUEZ-PULIDO et al., 2018). Fruto del interés por mejorar la calidad del profesorado universitario europeo, son propuestas de mejora como el informe Eurydice para la Modernización de la Educación superior en Europa: Personal Académico - 2017 (EUROPEAN COMMISSION/EACEA/EURYDICE, 2017).

El último informe presentado por la CRUETIC sobre la inclusión de la tecnología en las universidades españolas muestra una panorámica diferentes "dimensiones" que servirán de variables, a la hora de analizar cada apartado (ORTEGA et al., 2019, p. 11-12). Para ello, se han tenido en cuenta diferentes aspectos: metodológico, tecnológico, de contenidos y gestión. Uno de los descriptores más relacionados con el presente estudio es precisamente la Competencia digital (G1), que aparece mencionado dentro de la dimensión metodológica. Para medirla se pregunta a los docentes sobre el tipo de formación que reciben y en qué grado retorna esa formación a la institución en forma de proyectos de innovación docente. Asimismo, deben indicar en qué grado los estudiantes están aceptando este tipo de innovación en el aula y si conlleva algún tipo de reconocimiento posterior de forma que se incentiven estas prácticas. Resulta reseñable constatar el hecho de que a pesar de ser las "herramientas colaborativas" el tema más recurrente a la hora de organizar cursos de formación, con un total de 46 respuestas (apartado 14), su uso haya recibido un total de 54 respuestas (apartado 66.1), sin llegar a indicar en qué grado se les están dando uso real. Respecto al uso comunicación inmediata, en la mayoría de los casos no parece haber un sistema institucional identificado (apartado 66.2). El manejo de estas dos herramientas anteriormente mencionadas se hace muy necesario a la hora de trabajar de forma grupal, tal y como demandan los planes de Bolonia (DE VINCENZI, 2012; GIRONA; GUÀRDIA; MAS, 2018; MARTÍN GALÁN; RODRÍGUEZ MATEOS, 2012; PINEDA HERRERO; FERNÁNDEZ SEGUÍ, 2019), más aún en un contexto de enseñanza a distancia,

5 Participantes: UNED (España), Organización de Estados Iberoamericanos (OEI-Panamá) y Convenio Andrés Bello (CAB). 
algo que, debido a la crisis del Covid-19, se está extendiendo a universidades presenciales (ENSEÑAR..., 2020). El hecho de haber incorporado la tecnología en el aula previamente a este momento propiciará, en gran medida, el éxito de las mismas con la adquisición incluso de nuevos contenidos. Es interesante observar cómo los planes de formación se suelen ofrecer de manera voluntaria al PDI, a diferencia de otros planes diseñados para profesorado de enseñanza pre-universitaria. Además, suelen estar centrados en la docencia, dejando relegados otros aspectos como la gestión (PRENDES ESPINOSA; PORLÁN; SÁNCHEZ, 2018; RODRÍGUEZ-PULIDO; ARTILES-RODRIGUEZ; AGUILAR PERERA; ÁLAMO-BOLAÑOS, 2018).

\section{Descriptores del primer y segundo área competencial del DigComp según las diferentes actualizaciones}

La primera área que se ha estado trabajando por ser la primera del DigComp ha sido la de la Alfabetización Informacional. Esta se compone de tres competencias basadas en las seis iniciales propuestas por la UNESCO para la misma área (VUORIKARI; PUNIE, 2016, p. 35). Este documento añade además ejemplos de uso y dentro de estos ejemplos aparece el sector educativo, que a su vez debe seguir una serie de pasos, hasta llegar a la certificación. En niveles de educación primaria y secundaria, el tema se ha centrado en cómo localizar información de cara a preparar una clase o a que los estudiantes sean capaces de localizar sus propios recursos, ya que ahora los alumnos en estos niveles deben ser capaces de realizar sus búsquedas, aunque no sean del todo bibliográficas (INTEF, 2017). Es importante destacar que el Instituto Nacional de Tecnologías Educativas y de Formacion del Professorado (INTEF) basó su portfolio en la última actualización del DigComp (CARRETERO; VUORIKARI; PUNIE, 2017b), ya que incorporaba ocho niveles distintos de desempeño (aunque no se ajustó a ellos) y dos ejemplos de uso: uno en el entorno laboral, y otro en el educativo, aludiendo al tipo de habilidades que debería adquirir o conocer el usuario en cada uno de ellos. De esta forma, al adaptar los diferentes niveles al contexto universitario se deberían tener en consideración ambos entornos, de forma que la labor docente se vea completada con otras no incluidas en la docencia preuniversitaria, como podría ser la investigación. En este sentido, el interés por la formación en alfabetización informacional viene precedida por la digitalización de la biblioteca universitaria y otros centros de documentación (GUO, 2010; MALIK, 2013; MELO; DA SILVA, 2013), cuyo personal ha pasado a ser parte 
más activa en todas las titulaciones impartiendo sus propios cursos en los últimos cursos de los grados y másteres (HERNÁNDEZ; ERBEZ, 2012). En el caso de los NOOC que aquí se tratan, han sido los propios docentes con la colaboración del personal de la biblioteca, los que han creado e impartido los materiales para las competencias del área (navegación, evaluación y almacenamiento de la información).

Dicho esto, es importante tener en cuenta que no se debe ser competente en áreas estancas, sino que se debe ser capaz de aunar todas ellas de manera transversal, como por ejemplos, gestionar referencias bibliográficas en espacios de trabajo colaborativos (NICOL; LITTLEJOHN; GRIERSON, 2005). Esto debe ser además aplicable al propio profesorado, a estos con sus alumnos y a la cooperación entre estos mediante la realización de trabajos grupales, siendo más necesario aún en entornos de educación a distancia.

A medida que se va avanzando en las diferentes áreas competenciales se puede observar cómo el grado de detalle disminuye, producido quizá por tratarse de herramientas más específicas y cambiantes como son las que componen los contenidos la colaboración y la comunicación. Esto quiere decir que para impartir una formación actual y que perdure el mayor tiempo posible es necesario realizar una revisión continua de las mismas, llegando a modificar incluso los descriptores iniciales. Las competencias que componen este área son seis, y provienen de la descomposición de las tres iniciales que proponía la UNESCO en 2013 (VUORIKARI; PUNIE, 2016, p. 35).

\section{Metodología}

Uno de los objetivos de este estudio es hacer una propuesta metodológica de formación en las primeras dos áreas competenciales del DigComp a las necesidades propias del profesorado universitario. Dada la complejidad de la tarea, que depende a su vez de las necesidades metodológicas y tecnológicas de cada momento, este estudio ha seguido una metodología basada en la Investigación-Acción (PUTMAN; ROCK, 2017; REASON; BRADBURY, 2008). De este modo, el docente se convierte en investigador de su propio proceso, permitiendo que cada edición de los cursos NOOC se vaya mejorando cualitativamente con la implementación de las mejoras de los errores detectados en la inmediatamente que le precede. El siguiente gráfico muestra la evolución de dichas mejoras. De esta forma se ha pasado de la puesta en marcha de cursos independientes (en cierto modo) a la última fase que incorpora la certificación 
completa de toda un área competencial atendiendo a siempre a las tres funciones básicas del profesorado universitario: docencia, investigación y gestión.

\section{FIGURA 1 - EVOLUCIÓN DE LA INVESTIGACIÓN-ACCIÓN EN LA CONSECUCIÓN DE LOS CURSOS}

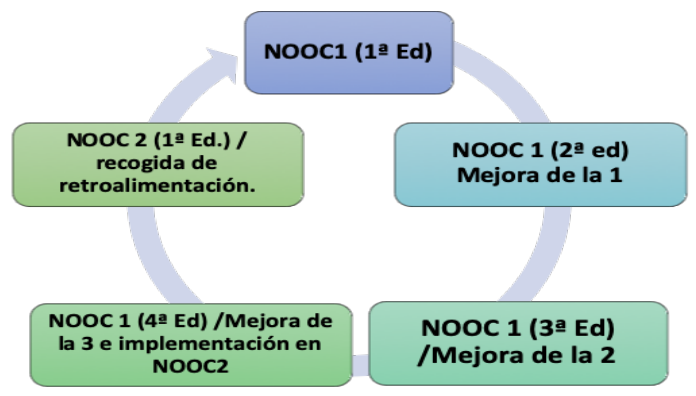

FUENTE: Elaboración propia

A tenor de lo expuesto, surgen varias preguntas de investigación: ¿En qué grado se corresponden las expectativas de estos cursos con lo que los matriculados terminan adquiriendo? ¿Realmente el contenido de los cursos resulta de utilidad al profesorado universitario, que es el foco principal de los mismos? En caso contrario, ¿Cuál podría ser la razón?

Para dar respuesta a estas preguntas, el equipo investigador se ha valido de diferentes herramientas de recogida de datos. En primer lugar, las pruebas de diagnóstico localizadas al principio de cada curso (competencia), seguidas de las encuestas iniciales y finales de tipo mixto y la observación directa mediante las herramientas de monitorización de la plataforma. Esto queda completado con la retroalimentación recibida a través de los foros y el correo electrónico tanto del equipo docente como de la gestión administrativa.

\section{Propuesta metodológica}

A continuación, se describen los elementos que conforman la estructura de los cursos propuestos para las áreas competenciales objeto de nuestro estudio: Información y Alfabetización informacional (NOOC1) y Comunicación y colaboración (NOOC2).

En primer lugar, y como instrumento de evaluación del participante, subrayamos la posibilidad de autoevaluar el nivel de dominio de la competencia 
a través de un cuestionario previo a la inscripción. Dependiendo de los resultados obtenidos, se recomendará al usuario matricularse en un nivel específico. Además, su realización le permitirá conocer en primera persona cuáles son sus deficiencias, potencialidades y conocimientos de los que ya dispone, a la vez que obtiene una imagen de sus necesidades, para construir su proceso de aprendizaje. En cuanto a las acciones, podrá llevar a cabo su proceso aprendizaje flexible configurando su proceso de aprendizaje personalizado y definiendo su plan de estudio en cuanto al nivel de dificultad que quiera afrontar. A todo esto, se añade la posibilidad de adquirir las correspondientes credenciales y acreditaciones derivadas de cada área competencial.

En segundo lugar, cabe mencionar la inclusión de dos encuestas de corte cuantitativo que aportan datos estadísticos de tipo general de interés a la hora de estudiar el alcance de los mismos. Estos nos permiten confirmar si efectivamente se están cumpliendo las expectativas de los usuarios y calibrar sus necesidades año tras año, a la vez que nos confirman si efectivamente el tipo de contenidos que se ha diseñado les resulta de utilidad para sus propios intereses.

A continuación, se muestra un gráfico de la estructura general de los elementos de recogida de datos y mejora de la calidad anteriormente mencionados para que ayude a comprender de una manera más completa la estructura de los propios cursos:

FIGURA 2 - REPRESENTACIÓN DEL PROCESO DE DESARROLLO DE LA PROPUESTA FORMATIVA

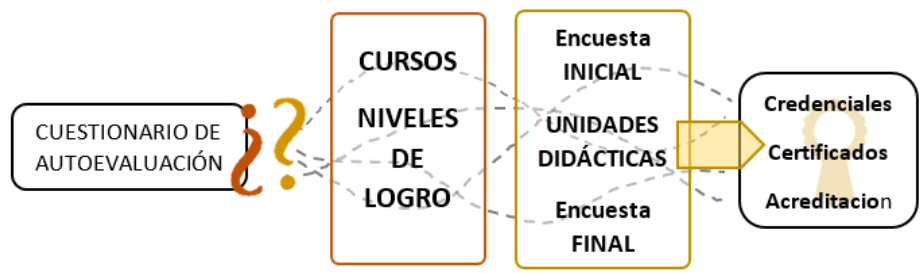

FUENTE: Elaboración propia.

Tal y como se puede observar en el anterior gráfico, los cursos desarrollados cuentan con una estructura que se repite en todos y cada uno de los mismos (PÉREZ SÁNCHEZ; JORDANO DE LA TORRE; MARTÍN CUADRADO, 2017, p. 12-13) en la que se contemplan diferentes elementos que buscan ofrecer al participante una visión global y concreta de cada contenido, objetivo, evaluación 
y logros obtenidos. Esta estructura común a cada área competencial se materializa para cada una de ellas mediante su plasmación en los siguientes gráficos:

\section{FIGURA3 - PRIMERAPROPUESTAPARA LAFORMACIÓN DE LA COMPETENCIA INFORMACIÓN Y ALFABETIZACIÓN INFORMACIONAL}

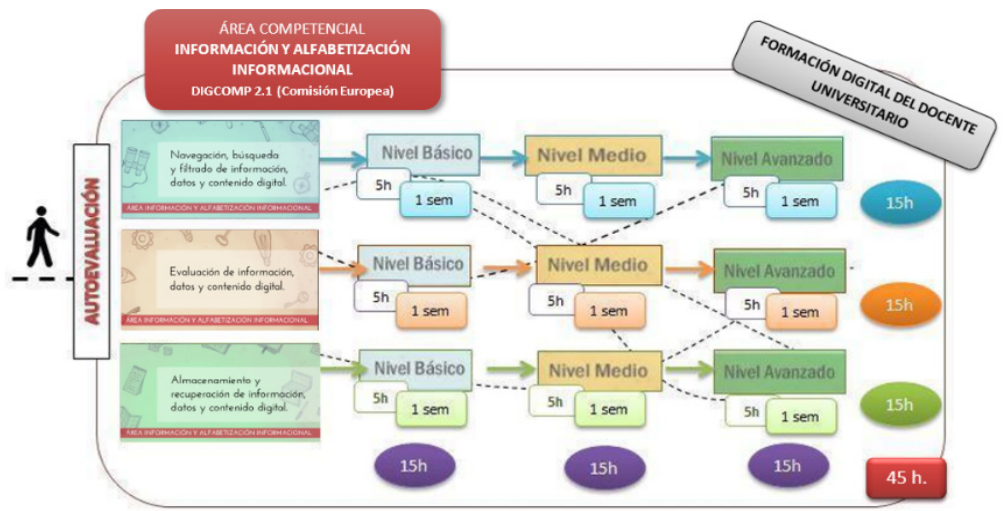

FUENTE: Elaboración propia.

Tal y como se ha mencionado anteriormente, gracias a los resultados obtenidos en los cuestionarios de diagnóstico, el usuario puede trazarse su propio plan de aprendizaje. Esto quiere decir una persona puede optar por realizar cursos sueltos de cada competencia, obtener la credencial de una o más competencias o recibir la certificación de toda un área competencial independientemente de que haya cursados todos los NOOC o no. Es importante destacar el hecho que esta estructura ha estado condicionada en todo momento por diferentes variables: el DigComp, la base normativa-tecnológica de UNED-Abierta (sustentada por Open Edx), y la literatura generada en tornos a los micro-créditos (AGUADED; MEDINA-SALGUERO, 2016; COAKLEY; GARVEY; O’NEILL, 2016; RYERSE, 2017).

Como ya se ha comentado con anterioridad, fue la cuarta edición la que incorporó la credencial por curso/competencia, además de la certificación de toda el área. Si bien la obtención del primero se basa en la contestación de 20 preguntas de tipo test como eje principal de todo un MOOC, la certificación del área exige la redacción de un caso práctico que demuestre que el profesor universitario o candidato es capaz de conjugar las tres competencias en una aplicación directa a su propio contexto profesional. Además, cada participante evaluará mediante revisión por pares el trabajo de otro compañero, una serie de criterios de evaluación disponible en la plataforma para todos los participantes.

Una vez puesta en marcha y a la vez que se recogían datos de la cuarta edición de la primera área competencial, se decidió replicar el mismo tipo de 
distribución estructural en la área de la Comunicación y la Colaboración, tal y como se muestra en la siguiente figura:

\section{FIGURA 4 - REPRESENTACIÓN DE LA PROPUESTA PARA LAFORMACIÓN DE} LA COMPETENCIA DE COMUNICACIÓN Y COLABORACIÓN

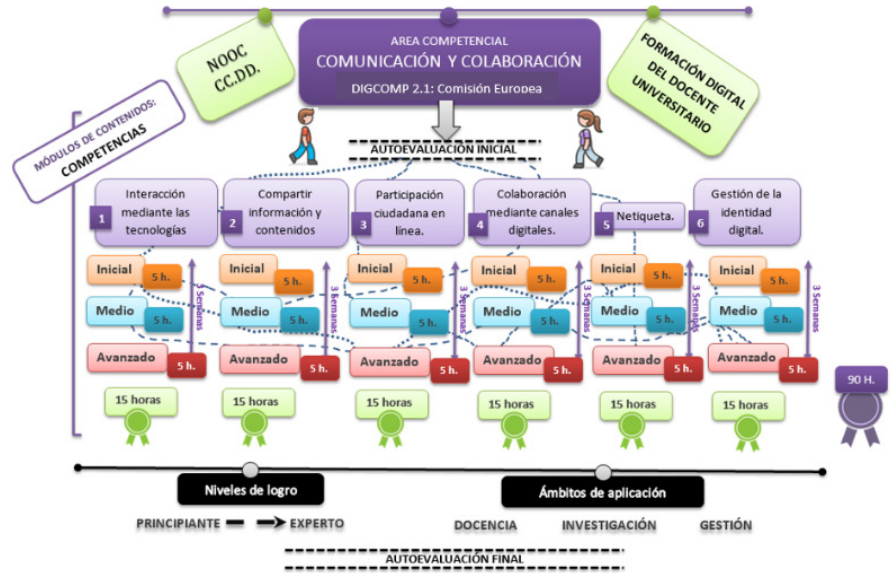

FUENTE: Elaboración propia.

Como se puede apreciar en el gráfico, además de los elementos diferenciadores de nuestra propuesta, se ha incorporado un aspecto nuevo y que se ha comenzado a tener en cuenta en esta primera edición del NOOC sobre Comunicación y Colaboración. Este elemento es el relativo a los ámbitos de aplicación, que aunque ya estaba presente en los NOOC1, ha sido en el gráfico de los NOOC2 cuando se ha hecho visible. Esta cuestión se ha venido a unir a nuestras consideraciones a la hora de diseñar los cursos y desarrollar nuestra propuesta, las cuales se han orientado a tres ámbitos de aplicación, la docencia, la investigación y la gestión. De esta forma, queda patente la diferente de estos cursos frente a otros dirigidos a otros docentes no relacionados con el ámbito universitario.

$\mathrm{Al}$ igual que en los cursos anteriores, cada curso sigue teniendo una carga de cinco horas de trabajo (que podría variar dependiente de la formación inicial del usuario), de tal forma que una competencia completa equivale a 15 horas de trabajo y el certificado final serían 90, ya que además de la adquisición de contenidos incorpora trabajo personal de investigación por parte del alumno. Aunque los cursos de acreditación de la competencia no figuren como tal en el gráfico equivalen a 5 horas, que incluye la lectura del material complementario de la temática correspondiente y el tiempo empleado en repasar todo lo aprendido en los cursos básico, medio y avanzado. El siguiente gráfico detalla más este aspecto. 
FIGURA 5 - REPRESENTACIÓN DEL PROCESO DE CONSECUCIÓN DE LAS CREDENCIALES Y EL CERTIFICADO DE LA COMPETENCIA DE LA NAVEGACIÓN, BÚSQUEDA Y FILTRADO DE INFORMACIÓN, DATOS Y CONTENIDO DIGITAL

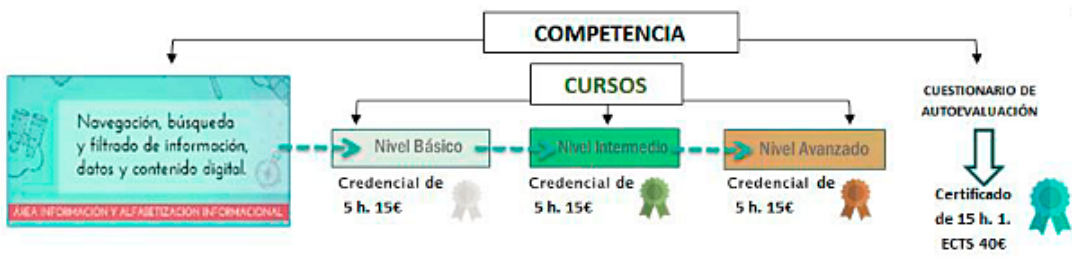

FUENTE: Elaboración propia.

Como ya se ha indicado al comienzo de este documento, para realizar el diseño de este sistema de certificaciones, se ha seguido en todo momento las directrices marcadas por UNED-Abierta y la normativa de la propia institución. 


\section{Análisis de datos}

El siguiente apartado muestra una selección de los datos más relevantes obtenidos mediante las diferentes fuentes descritas en relación al área competencial de la Alfabetización Informacional. Con relación a la matriculación, el dato más relevante tiene que ver con el nivel de desempeño, ya que el grueso se sitúa en el básico a vez que el número decae a media de que se suceden las ediciones. La razón habría que encontrarla quizá en una tendencia general de este tipo de cursos masivos o en una deficiente difusión de estos cursos en particular. Este será uno de los focos de investigación en el futuro más inmediato.

FIGURA 6 - TABLA DEL NÚMERO DE PARTICIPANTES MATRICULADOS

\begin{tabular}{|c|c|c|c|}
\hline $\begin{array}{c}\text { Número de } \\
\text { matriculados/ } \\
\begin{array}{c}\text { Cuestionarios de } \\
\text { Autoevaluación }\end{array}\end{array}$ & Navegación & Evaluación & Almacenamiento \\
\hline Nivel básico & $1170 / 1085$ & $982 / 966$ & $1346 / 1314$ \\
\hline Nivel medio & $623 / 256$ & $583 / 170$ & $680 / 218$ \\
\hline Nivel avanzado & $697 / 183$ & $719 / 242$ & $769 / 266$ \\
\hline TOTALES & $2490 / 1524$ & $2284 / 1378$ & $2795 / 1798$ \\
\hline
\end{tabular}

FUENTE: Elaboración propia.

En relación a la perspectiva que los participantes tienen sobre su propio nivel de competencia en cada uno de los cursos, destacamos que la gran mayoría se perciben como "aspirantes" en un 55\%, un 64\% y un 58\%, de forma previa al comienzo de los cursos. Respecto a la edad de los participantes, la mayoría gira en torno a los 40 y 59 en todos los cursos del área, aunque el interés suscitado por los temas tratados se comparte mayoría de los grupos de edad. Estos, manifiestan ser conscientes de la necesidad de estar al día en tanto a formación digital se refiere. También podemos deducir que los dos intervalos de edad que sobresalen coinciden con las edades en las que los participantes están en pleno desempeño profesional y con necesidad de formarse para su mejor desarrollo laboral.

Respecto a la motivación, se aprecie el hecho de que la mayoría se han matriculado en el curso por sentirse atraídos por su temática, su metodología y su relación con su entorno profesional, además de otros aspectos. 
FIGURA 7 - TABLA SOBRE MOTIVACIONES DE LOS PARTICIPANTES PARA REALIZAR LOS CURSOS NOOC

\begin{tabular}{|l|c|c|c|}
\hline \multicolumn{1}{|c|}{$\begin{array}{c}\text { MOTIVACIONES PARA LA } \\
\text { REALIZACIÓN DE LOS NOOC }\end{array}$} & Navegación & Evaluación & Almacenamiento \\
\hline Me interesa la temática del curso & $\mathbf{4 9 9}$ & $\mathbf{1 7 1}$ & $\mathbf{1 8 3}$ \\
\hline La metodología del curso me resulta cómoda & $\mathbf{1 8 9}$ & $\mathbf{6 6}$ & $\mathbf{7 7}$ \\
\hline El curso es útil para conocer mejor la UNED & 42 & 21 & 25 \\
\hline El curso es útil para mi desarrollo profesional & $\mathbf{3 3 8}$ & $\mathbf{1 2 0}$ & $\mathbf{1 3 0}$ \\
\hline $\begin{array}{l}\text { El prestigio de la UNED me anima a realizar } \\
\text { un curso en esta institución }\end{array}$ & 149 & 57 & 48 \\
\hline $\begin{array}{l}\text { Me interesa tener acceso a contenidos concretos } \\
\text { del curso }\end{array}$ & 78 & 42 & 55 \\
\hline $\begin{array}{l}\text { Me interesa interactuar con otros estudiantes } \\
\text { con quienes comparto intereses }\end{array}$ & $\mathbf{2 4}$ & $\mathbf{1 3}$ & $\mathbf{1 3}$ \\
\hline $\begin{array}{l}\text { Me interesa tener acceso a profesorado } \\
\text { experto en la temática }\end{array}$ & $\mathbf{6 7}$ & $\mathbf{2 9}$ & $\mathbf{2 4}$ \\
\hline
\end{tabular}

FUENTE: Elaboración propia.

Sorprende el hecho que una gran mayoría espere completar el curso completo cuando finalmente los datos confirman que no ha sido así. Comparado con la siguiente tabla, los cursos parecen haber tenido un alto grado de aceptación de que aquellos que han conseguido terminar, con lo cual se desprende que una gran mayoría ha abandonado por falta del tiempo, al igual que ocurre con este tipo de curso en otros contextos.

FIGURA 8-TABLA SOBRE EXPECTATIVAS DE LOS PARTICIPANTES EN LOS NOOC

\begin{tabular}{|l|c|c|c|}
\hline \multicolumn{1}{|c|}{ EXPECTATIVAS SOBRE LOS NOOC } & Navegación & Evaluación & Almacenamiento \\
\hline $\begin{array}{l}\text { Mi intención es consultar los materiales, pero } \\
\text { no espero finalizar el curso }\end{array}$ & 17 & 2 & 1 \\
\hline $\begin{array}{l}\text { Espero realizar algunas de las actividades, } \\
\text { pero no creo que complete todo el curso }\end{array}$ & 31 & 9 & 11 \\
\hline Espero completar el curso completo & $\mathbf{5 5 6}$ & $\mathbf{1 9 0}$ & $\mathbf{2 0 3}$ \\
\hline
\end{tabular}

FUENTE: Elaboración propia.

Presentamos a continuación una tabla que recopila los datos de los tres cursos y de sus respectivos niveles de dificultad, en cuanto los diferentes elementos: expectativas del participante, contenidos del curso, metodología, duración, organización y estructura y funcionamiento de la plataforma virtual. 
FIGURA 9 - TABLA SOBRE LA PERCEPCIÓN DE LOS CURSOS Y SUS ELEMENTOS MÁS RELEVANTES

\begin{tabular}{|c|c|c|c|c|c|c|c|c|c|}
\hline & \multicolumn{3}{|c|}{ Navegación } & \multicolumn{3}{c|}{ Evaluación } & \multicolumn{3}{c|}{ Almacenamiento } \\
\hline & Básico & Medio & Avanzado & Básico & Medio & Avanzado & Básico & Medio & Avanzado \\
\hline $\mathbf{1}$ & $78,24 \%$ & $81,8 \%$ & $72,1 \%$ & $77,4 \%$ & $78,6 \%$ & $81,5 \%$ & $75,2 \%$ & $5 \%$ & $81 \%$ \\
\hline $\mathbf{2}$ & $76 \%$ & $79,2 \%$ & $72,1 \%$ & $76,1 \%$ & $77,1 \%$ & $84,6 \%$ & $73,6 \%$ & $5 \%$ & $78,2 \%$ \\
\hline $\mathbf{3}$ & $76,3 \%$ & $76,6 \%$ & $73,2 \%$ & $80,6 \%$ & $80,4 \%$ & $81,5 \%$ & $72,6 \%$ & $6 \%$ & $74,5 \%$ \\
\hline $\mathbf{4}$ & $79,6 \%$ & $76,6 \%$ & $72,4 \%$ & $81 \%$ & $78 \%$ & $81,5 \%$ & $77,4 \%$ & $7,5 \%$ & $85,5 \%$ \\
\hline $\mathbf{5}$ & $82 \%$ & $80,5 \%$ & $74,4 \%$ & $83,6 \%$ & $84 \%$ & $87 \%$ & $80,6 \%$ & $4,5 \%$ & $82,7 \%$ \\
\hline $\mathbf{6}$ & $89 \%$ & $80,5 \%$ & $83,8 \%$ & $90 \%$ & $86,4 \%$ & $91,5 \%$ & $86,4 \%$ & $4,8 \%$ & $91 \%$ \\
\hline
\end{tabular}

1.- El curso se ha ajustado a mis expectativas.

2.- Los contenidos del curso me han parecido adecuados y suficientes.

3.- La metodología del curso me ha resultado motivadora y apropiada.

4.- La duración del curso me ha parecido adecuada.

5.- El curso está bien organizado / estructurado.

6.- La plataforma virtual ha funcionado correctamente.

FUENTE: Elaboración propia.

Esto se completa con datos de otros ítems que constata que los niveles de satisfacción en general han sido superiores al $75 \%$, incluyendo algunos que han alcanzado casi el 90\%.

\section{Conclusiones}

Una vez descritos algunos de los resultados más relevantes de esta investigación, a continuación, se resumen las conclusiones que han arrojado una vez cerradas las cuatro ediciones los NOOC1 y que permitirán el avance de las siguientes ediciones tanto de estos como de los NOOC2. Contestando a las preguntas de investigación anteriormente formuladas, se puede afirmar que, en general, el nivel de expectativas de los participantes se ha cumplido de manera general, aunque la tasa de abandono ha sido elevada, tal y como suele suceder a otro tipo de formación similar (HENDERIKX; KREIJNS; KALZ, 2017; POY; GONZALES-AGUILAR, 2014; VÁZQUEZ CANO; LÓPEZ MENESES, 2015). Aunque los cursos han sido diseñados especialmente para el docente universitario, y así se percibe claramente en sus 46 contenidos, las diferentes ediciones continúan recibiendo docentes de otros ámbitos, así como 
profesionales de otros gremios. Quizá esta pueda ser una de las razones del escaso número de personas interesadas en conseguir la acreditación de las competencias y certificación del área competencial, contrariamente a lo que se esperaba. Es por ello que, uno de los retos sigue siendo captar al docente universitario para que sientan la necesidad de actualizar sus competencias digitales de cara a desarrollar mejor su tarea en la docencia, investigación y gestión.

Al largo del desarrollo de los cursos se han trabajado las herramientas más utilizadas en entornos docentes y de investigación, así como otras no tan conocidas que han sido todo un descubrimiento para muchos de participantes, como así lo han comentados mediante los foros o de forma privada a miembros del equipo docente. Estos contenidos les han permitido realizar búsquedas avanzadas en bases de datos científicas, distinguir fuentes de información fiable de la que no lo es y almacenar información de forma ordenada y segura. Sea como fuere, los contenidos han resultado del agrado de todos, aunque siguen necesitando la retroalimentación de sus usuarios para continuar el ciclo de mejoras marcado en la metodología. Muchas de las herramientas han sido tratadas conforme a versiones de hace cinco años y muchas de ellas han cambiado o simplemente han sido sustituidas por otras. Es por ello que urge la necesidad de realizar más material audiovisual adaptado a estos nuevos contenidos centrándose en los aspectos menos desarrollados del curso.

Situaciones de emergencia como las del Covid-19 han puesto en cuestionamiento la competencia digital del profesorado y de sus alumnos. Esto ha provocado un interés repentino por aprender a utilizar la tecnología de forma rápida y ser capaces de realizar tareas de aula en las casas de cada estudiante. En un intento de apoyo solidario, son muchos los proyectos y acciones que han salido a la luz para tratar de paliar las lagunas docentes más usuales. Se han puesto a disposición de los docentes tantos cursos y formación que, para ellos, en tan poco espacio de tiempo pueda resultar abrumador. Muchos de los recursos y técnicas contenidas en los NOOC1 y NOOC2 resultarían ahora eficaces para la situación de emergencia desatada, y es por ello que se han publicado ediciones especiales de las mismas. Este puede ser un buen momento para recoger sugerencias y aspectos de mejora de los materiales de cara ediciones futuras de ambos NOOC. 


\section{REFERENCIAS}

AGUADED, Ignacio; MEDINA-SALGUERO, Rosario. Certificación de los MOOC y su reconocimiento en créditos universitarios. International Studies on Law and Education, Porto, n. 23. p. 39-50, maio/ago. 2016. Disponible en: http:/www.hottopos.com/isle23/3950AguadedMedina.pdf. Acceso en: 21 agosto 2020.

ÁLVAREZ, Mabel et al. Aplicación de competencias digitales en un curso universitario. La historia de una experiencia. En: CONGRESO INTERNACIONAL DE TECNOLOGÍAS PARA LA EDUCACIÓN Y EL CONOCIMENTO, 24., 2019, Madrid. Actas [...]. Madrid: UNED, 2019. p. 381-391. Disponible en: http://e-spacio.uned.es/fez/eserv/bibliuned:424Mlcacheiro-0001/actas_interestratic2019_vol_3.pdf. Acceso en: 20 mayo 2020.

BUSTOS-GONZÁLEZ, Atilio. Tránsito de universidad docente a universidad de investigación. ¿un problema de información académica, de taxonomías o de rankings universitarios? El Profesional de La Información, Madrid, v. 28, n. 4, p. 1-14, 2019. Disponible en: https://doi.org/10.3145/epi.2019.jul.22. Acceso en: 15 abr. 2020.

CARRETERO GOMEZ, Stephane; VUORIKARI, Riina; PUNIE, Yves. DigComp 2.1: the digital competence framework for citizens with eight proficiency levels and examples of use. Luxembourg: Publication Office of the European Union, 2017a. Disponible en: https://doi.org/10.2760/38842. Acceso en: 18 abr. 2020.

CARRETERO GOMEZ, Stephane; VUORIKARI, Riina; PUNIE, Yves. The Digital Competence Framework for Citizens With eight proficiency levels and examples of use. Luxembourg: Publications Office of the European Union, 2017b. Disponible en: https:// doi.org/10.2760/38842. Acceso en: 21 agosto 2020.

COAKLEY, Darragh; GARVEY, Roisin; O’NEILL, Íde. Micro-learning - Adopting Digital Pedagogies to Facilitate Technology-Enhanced Teaching and Learning for CPD. En: TEH, Geok Bee; CHOY, Siew Chee (ed.). Empowering 21st Century Learners Through Holistic and Enterprising Learning. Malaysia: Springer Singapore, 2016. p. 237-242. Disponible en: https://doi.org/10.1007/978-981-10-4241-6_24. Acceso en: 1 mayo 2020.

EUROPEAN COMMISSION/EACEA/EURYDICE. Modernisation of Higher Education in Europe: Academic Staff. Eurydice Report. Luxembourg: Publications Office of the European Union, 2017. Disponible en: https://doi.org/10.2797/408169. Acceso en: 21 agosto 2020 .

DE VINCENZI, Ariana. La formacion pedagogica del profesor universitario. Un desafio para la reflexion y revision de la practica docente en el nivel superior. Aula, Salamanca, España, v. 18, p. 111-122, 2012. Disponible en: http://revistas.usal.es/index.php/02143402/article/view/8875. Acesso en: 12 mayo 2020. 
DURÁN CUARTERO, Marta; GUTIÉRREZ PORLÁN, Isabel. Diseño y validación de un instrumento de evaluación para la certificación de la competencia TIC del profesorado universitario. Revista Mexicana de Investigación Educativa, México, v. 21, n. 69, p. 527-556, abr./jun. 2016. Disponible en: https://doi.org/10.13140/2.1.4232.4165. Acesso en: 18 abr. 2020.

ENSEÑAR y aprender en linea. Conectad@s: la universidad en casa, 2020. Disponible en: https://www.uned.es/universidad/inicio/uned_uoc_solidaria/elearning.html. Acceso en: 10 abr. 2020.

FERRARI, Anusca. DIGCOMP: A Framework for Developing and Understanding Digital Competence in Europe. En: PUNIE, Yves; BRECKO, Barbara N. (ed.). Joint Reseach Centre, European Comission. Luxembourg: Publications Office of the European Union, 2013. Disponible en: https://doi.org/10.2788/52966. Acesso en: 20 mayo 2020.

GIRONA, Cristina; GUÀRDIA, Lourdes; MAS, Xavier. La Docencia Universitaria más allá del 2020: tendencias, retos y nuevos escenarios. En: CARRASCO, Salvador; CORRAL, Ignacio Del (coord.). Docencia Universitaria e Innovación. Evolución y Retos a través de los CIUDU. Barcelona: Octaedro, 2018. p. 195-226.

GUO, Linghui. On construction of digital libraries in universities. En: INTERNATIONAL CONFERENCE ON COMPUTER SCIENCE AND INFORMATION TECHNOLOGY, 3RD., 2010, Chengdu, China, Annals [...]. Chengdu, China: IEEE, 2010. p. 452-456. Disponible en: https://ieeexplore.ieee.org/abstract/document/5564750. Acceso en: 21 agosto 2020 .

HENDERIKX, Maartze A.; KREIJNS, Karel; KALZ, Marco. Refining success and dropout in massive open online courses based on the intention-behavior gap. Distance Education, Australia, v. 38, n. 3, p. 353-368, 2017. Disponible en: https://doi.org/10.10 80/01587919.2017.1369006. Acesso en: 20 mayo 2020.

HERNÁNDEZ, Carmen Julia; ERBEZ, José Manuel. La formación en competencias de la biblioteca de la universidad de la laguna en el contexto de la cooperación universitaria. Revista Española de Documentacion Cientifica, España, n. 35, p. 169-188, 2012. (MONOGRAFICO). Disponible en: https://doi.org/10.3989/redc.2012.mono.981. Acesso en: 20 abr. 2020.

INTEF (Instituto Nacional de Tecnologías Educativas y de Formacion del Professorado). Marco Común de Competencia Digital Docente. Madrid, Sep. 2017. Disponible en: https://aprende.intef.es/sites/default/files/2018-05/2017_1020_Marco-Com\%C3\%BAnde-Competencia-Digital-Docente.pdf. Acceso en: 20 mayo 2020.

JORDANO DE LA TORRE, María; MARTÍN CUADRADO, Ana María; PÉREZ SÁNCHEZ, Lourdes. Adapting the DigComp - descriptors-to the University teaching $\&$ researching needs through NOOCs Outline. En: THE ONLINE, OPEN AND FLEXIBLE HIGHER EDUCATION CONFERENCE, 2019, Madrid. Papers [...]. Madrid: OOFHEC, 2019. Disponible en: https://doi.org/10.13140/RG.2.2.34678.96328. Acceso en: 21 ago. 2020. 
MALIK, Anita. Digitalization and Automation in University Libraries. IOSR Journal Of Humanities And Social Science, [s. l.], v. 8, n. 1, p. 60-64, jan./feb. 2013.

MARTÍN GALÁN, Bonifacio; RODRÍGUEZ MATEOS, David. La evaluación de la formación universitaria semipresencial y en línea en el contexto del EEES mediante el uso de los informes de actividad de la plataforma Moodle. RIED: Revista Iberoamericana de Educación a Distancia, Madrid, v. 15, n. 1, p. 159-178, 2012.

MAUREL, Maria del Carmen; CUENCA-PLETSCH, Liliana; DALFARO, Nidia. Incorporación de nuevas estrategias en el aula. Relación entre el impacto académico y el rol asumido por el docente. Ciencia y Tecnología, Chaco, Argentina, n. 13, p. 337-349, 2013.

MELO, Amanda Meincke; SILVA, Joseane Giacomelli da. Online digital libraries at universities: an inclusive proposal. En: STEPHANIDIS, Constantine; ANTONA, Margherita (ed.). Universal Access in Human-Computer Interaction. Applications and Services for Quality of Life. International Conference on Universal Access in HumanComputer Interaction, 7 th. Lecture Notes in Computer Science. Berlin: Springer, 2013. Disponible en: https://doi.org/10.1007/978-3-642-39194-1_44. Acceso en: 21 agosto 2020.

MONEREO, Carles; DOMÍNGUEZ, Carola. La Identidad docente de los Profesores Universitarios Competentes. Educación XX1, Madrid, v. 17, n. 2, p. 83-104, 2014.

NICOL, David; LITTLEJOHN, Allison; GRIERSON, Hilary. The importance of structuring information and resources within shared workspaces during collaborative design learning. Open Learning, London, v. 20, n. 1, p. 31-49, 2005.

ORTEGA, Juan Gomez et al. Informe de la situación de las tecnologías educativas en las universidades españolas 2018. España: CRUE, 2019.

PRENDES ESPINOSA, Maria Paz; PORLÁN, Isabel Gutiérrez; SÁNCHEZ, Francisco Martínez. Digital competence: A need for university teachers in the 21 st century. Revista de Educacion a Distancia, Murcia, n. 56, p. 1-22, 2018. Disponible en: https://doi. org/10.6018/red/56/7. Acceso en: 20 mayo 2020.

PÉREZ SÁNCHEZ, Lourdes; JORDANO DE LA TORRE, Maria; MARTÍN CUADRADO, Ana María. Los NOOC para la formación en competencias digitales del docente universitario. Una experiencia piloto de la Universidad Nacional de Educación a Distancia (UNED). NOOCs for the development of university teachers digital competences. A pilot experien. RED. Revista de Educación a Distancia, Murcia, n. 55, p. 1-35, 2017. Disponible en: https://doi.org/http://dx.doi.org/10.6018/red/55/1. Acceso en: 20 abr. 2020.

PINEDA HERRERO, Pilar; FERNÁNDEZ SEGUÍ, Héctor. Cómo evaluar el impacto de la educación continua. Retos y Claves de la Educación Continua. Red de Educación Continua de Latinoamérica y Europa, Bogotá, n. 6, p. 34-49, 2019.

POY, Raquel; GONZALES-AGUILAR, Audilio. Factores de exito de los MOOC: algunas consideraciones criticas. RISTI - Revista Iberica de Sistemas e Tecnologias de Informacao, Coimbra, n. E1, p. 105-118, marzo 2014. Disponible en: https://doi.org/10.4304/risti. e1.105-118. Acceso en: 20 abr. 2020. 
REDECKER, Christine. Proposal for a European Framework for the Digital Competence of Educators: DigCompEdu. PUNIE, Yves (ed.). Luxembourg: Publications Office of the European Union, 2017. Disponible en: http://publications.jrc.ec.europa.eu/repository/ handle/JRC107466. Acceso en: 21 agosto 2020.

RYERSE, Mary. Competency-Based Micro-credentials are Transforming Professional Learning. In: Getting Smart, [s.l.], 2017. Disponible em: https://www.gettingsmart. com/2017/11/micro-credentials-transforming-professional-learning/. Acceso en: 21 nov. 2019.

PUTMAN, S. Michael; ROCK, Tracy. C. Action Research: Using Strategic Inquiry to Improve Teaching and Learning. Los Angeles: SAGE Publication, 2017.

REASON, Peter; BRADBURY, Hilary. The SAGE handbook of action research: participative inquiry and practice. Los Angeles: SAGE, 2008.

RODRÍGUEZ-PULIDO, Josefa et al. Formación permanente para el docente universitario: Enfoques y propuestas. Docencia e Investigación, Toledo, Espanã, n. 28, p. 27-49, 2018.

UNITED NATIONS. UNESCO ICT Competency Framework for Teachers (2.0). París: UNESCO \& Microsoft, 2011.

VÁZQUEZ CANO, Esteban; LÓPEZ MENESES, Eloy. La filosofía educativa de los MOOC y la educación universitaria. RIED: Revista Iberoamericana de Educación a Distancia, Madrid, v. 18, n. 2, p. 25-37, 2015.

VUORIKARI, Riina et al. DigComp 2.0: The Digital Competence Framework for Citizens. Update Phase 1: the Conceptual Reference Model. European Commission. Luxembourg: Publication Office of the European Union, 2016. Disponible en: https:// doi.org/10.2791/11517. Acceso en: 21 agosto 2020.

WELlER, Saranne. Academic Practice: Developing as a Professional in Higher Education. London: SAGE, 2019.

Recibido en $15 / 07 / 2020$.

Aprobado en 24/08/2020. 